\title{
THE COMMENSURABILITY RELATION FOR FINITELY GENERATED GROUPS
}

\author{
SIMON THOMAS
}

AbstraCt. There does not exist a Borel way of selecting an isomorphism class within each commensurability class of finitely generated groups.

\section{INTRODUCTION}

Two $G_{1}, G_{2}$ are said to be (abstractly) commensurable, written $G_{1} \approx_{C} G_{2}$, iff there exist subgroups $H_{i} \leqslant G_{i}$ of finite index such that $H_{1} \cong H_{2}$. In this paper, we shall consider the commensurability relation on the space $\mathcal{G}$ of finitely generated groups. Here $\mathcal{G}$ denotes the Polish space of finitely generated groups introduced by Grigorchuk [4]; i.e. the elements of $\mathcal{G}$ are the isomorphism types of marked groups $\langle G, \bar{c}\rangle$, where $G$ is a finitely generated group and $\bar{c}$ is a finite sequence of generators. (For a clear account of the basic properties of the space $\mathcal{G}$, see either Champetier [1] or Grigorchuk [6].) Our starting point is the following result, which inituitively says that the isomorphism and commensurability relations on $\mathcal{G}$ have exactly the same complexity.

Theorem 1.1. The isomorphism relation $\cong$ and commensurability relation $\approx_{C}$ on the space $\mathcal{G}$ of finitely generated groups are Borel bireducible.

In particular, there exists a Borel reduction from $\approx_{C}$ to $\cong$; i.e. a Borel map $f: \mathcal{G} \rightarrow \mathcal{G}$ such that if $G, H \in \mathcal{G}$, then

$$
G \approx_{C} H \quad \text { iff } \quad f(G) \cong f(H) .
$$

However, while the proof of Theorem 1.1 shows that there exists a Borel reduction from $\approx_{C}$ to $\cong$, it does not provide an explicit example of such a reduction. (On the other hand, as we shall see in Section 2, it is straightforward to find an explicit example of a Borel reduction from $\cong$ to $\approx_{C}$.) 
Open Problem 1.2. Find an explicit "group-theoretic" Borel reduction $f: \mathcal{G} \rightarrow \mathcal{G}$ from the commensurability relation $\approx_{C}$ to the isomorphism relation $\cong$.

Of course, one approach to this problem would be to seek an explicit "grouptheoretic" Borel map which selects an isomorphism class within each commensurability class of finitely generated groups. However, the main result of this paper shows that no such map exists.

Theorem 1.3. There does not exist a Borel reduction $f: \mathcal{G} \rightarrow \mathcal{G}$ from $\approx_{C}$ to $\cong$ such that $f(G) \approx_{C} G$ for all $G \in \mathcal{G}$.

This paper is organized as follows. In Section 2, after recalling some of the basic theory of countable Borel equivalence relations, we shall present the proof of Theorem 1.1. In Section 3, after a short discussion of the branch groups constructed in Segal [11], we shall present the proof of Theorem 1.3. Finally, in Section 4, we shall consider the analogs of Theorem 1.3 for the virtual isomorphism and quasiisometry relations on the space $\mathcal{G}$ of finitely generated groups.

\section{Countable Borel equivalence Relations}

In this section, we shall present the proof of Theorem 1.1. But first we need to recall some of the basic theory of countable Borel equivalence relations.

Let $X$ be a standard Borel space; i.e. a Polish space equipped with its associated $\sigma$-algebra of Borel subsets. Then a Borel equivalence relation on $X$ is an equivalence relation $E \subseteq X^{2}$ which is a Borel subset of $X^{2}$. If $E, F$ are Borel equivalence relations on the standard Borel spaces $X, Y$ respectively, then we say that $E$ is Borel reducible to $F$ and write $E \leq_{B} F$ if there exists a Borel map $f: X \rightarrow Y$ such that $x E y$ iff $f(x) F f(y)$. Such a map $f$ is called a Borel reduction from $E$ to $F$. We say that $E$ and $F$ are Borel bireducible and write $E \sim_{B} F$ if both $E \leq_{B} F$ and $F \leq_{B} E$. Finally we write $E<_{B} F$ if both $E \leq_{B} F$ and $F \leq_{B} E$.

In this paper, we shall mainly be concerned with countable Borel equivalence relations; i.e. Borel equivalence relations $E$ such that every $E$-equivalence class is countable. A detailed development of the general theory of countable Borel equivalence relations can be found in Jackson-Kechris-Louveau [9]. Here we shall only recall some of the most basic results of the theory. 
With respect to Borel reducibility, the least complex countable Borel equivalence relations are those which are smooth; i.e. those countable Borel equivalence relations $E$ on a standard Borel space $X$ for which there exists a Borel function $f: X \rightarrow Y$ into a standard Borel space $Y$ such that $x E y$ iff $f(x)=f(y)$. Equivalently, the countable Borel equivalence relation $E$ on $X$ is smooth iff the quotient $X / E$ is a standard Borel space. Next in complexity come those countable Borel equivalence relations $E$ which are Borel bireducible with the Vitali equivalence relation $E_{0}$, which is defined on the space $2^{\mathbb{N}}$ of infinite binary sequences by

$$
x E_{0} y \quad \text { iff } \quad x(n)=y(n) \text { for all but finitely many } n .
$$

More precisely, by Harrington-Kechris-Louveau [8], if $E$ is any (not necessarily countable) Borel equivalence relation, then $E$ is nonsmooth iff $E_{0} \leq_{B} E$. It turns out that there is also a most complex countable Borel equivalence relation $E_{\infty}$, which is universal in the sense that $F \leq_{B} E_{\infty}$ for every countable Borel equivalence relation $F$. (Clearly this universality property uniquely determines $E_{\infty}$ up to Borel bireducibility.) $E_{\infty}$ has a number of natural realisations in many areas of mathematics, including algebra, topology and recursion theory. In particular, by Thomas-Velickovic [14], the isomorphism relation $\cong$ on the space $\mathcal{G}$ of finitely generated groups is a universal countable Borel equivalence.

Proof of Theorem 1.1. It is well-known that if $G$ is a finitely generated group, then there exist only countably many groups $H$ up to isomorphism such that $G \approx_{C} H$. (For example, see Erschler [2].) It follows that the commensurability relation $\approx_{C}$ is a countable Borel equivalence relation on the space $\mathcal{G}$ of finitely generated groups. Since the isomorphism relation is a universal countable Borel equivalence, it follows that $\left(\approx_{C}\right) \leq_{B}(\cong)$.

In order to see that $(\cong) \leq_{B}\left(\approx_{C}\right)$, let $S$ be a fixed infinite finitely generated group and consider the Borel map $h: \mathcal{G} \rightarrow \mathcal{G}$ defined by

$$
G \stackrel{h}{\mapsto}(\operatorname{Alt}(5) \text { wr } G) \text { wr } S .
$$

(Here wr denotes the restricted wreath product.) Combining Lemma 2.2(b) and Theorem 2.5 of Thomas [12], we obtain that if $G, H$ are arbitrary groups, then

$$
G \cong H \quad \text { iff } \quad(\operatorname{Alt}(5) \operatorname{wr} G) \operatorname{wr} S \approx_{C}(\operatorname{Alt}(5) \text { wr } H) \operatorname{wr} S .
$$


In particular, $h$ is a Borel reduction from $\cong$ to $\approx_{C}$.

Remark 2.1. Strictly speaking, in the proof of Theorem 1.1, we should first fix some finite sequences $\bar{a}, \bar{b}$ of generators for $\operatorname{Alt}(5), S$ respectively and then let $h$ be the Borel map on the space $\mathcal{G}$ of marked finitely generated groups defined by

$$
\langle G, \bar{c}\rangle \stackrel{h}{\mapsto}\left\langle(\operatorname{Alt}(5) \text { wr } G) \text { wr } S, \bar{a}^{\wedge} \bar{b}^{\wedge} \bar{c}\right\rangle \text {. }
$$

Having done this, it is easily checked that $h$ is actually a continuous reduction from $\cong$ to $\approx_{C}$

On the other hand, the above argument for the existence of a Borel reduction $f: \mathcal{G} \rightarrow \mathcal{G}$ from $\approx_{C}$ to $\cong$ ultimately relies on the Feldman-Moore Theorem [3] that every countable Borel equivalence relation can be realized as the orbit equivalence relation of a Borel action of some countable group; and this argument provides no information concerning the Borel complexity of such a reduction.

Question 2.2. Does there exist a continuous reduction $f: \mathcal{G} \rightarrow \mathcal{G}$ from the commensurability relation $\approx_{C}$ to the isomorphism relation $\cong$ ?

\section{Some Finitely GENERATED BRANCH GROUPS}

In this section, we shall present the proof of Theorem 1.3. But first we need to recall some of the basic notions from the theory of branch groups. (A detailed account of the theory can be found in Grigorchuk [5].)

Let $\left(\ell_{n}\right)_{n \geq 0}$ be a sequence of integers such that $\ell_{n} \geq 2$ for all $n$. Then the rooted tree of type $\left(\ell_{n}\right)_{n \geq 0}$ is a tree $T$ with a distinguished vertex $v_{0}$ (called the root) of valency $\ell_{0}$ such that every vertex at distance $n \geq 1$ from $v_{0}$ has valency $\ell_{n}+1$. The distance from $v_{0}$ to a vertex $v$ is called the level of $v$ and the set of vertices of level $n$ is called the $n$th layer of $T$. If we picture the tree $T$ with $v_{0}$ at the top and with $\ell_{n}$ edges descending from each vertex of level $n$, then each vertex $v$ of level $m$ is the root of a rooted subtree $T_{v}$ of $T$ of type $\left(\ell_{n}\right)_{n \geq m}$.

Let $G$ be a subgroup of $\operatorname{Aut}(T)$. Then for each vertex $v$, the corresponding rigid stabilizer $\operatorname{rist}_{G}(v)$ is the subgroup of $G$ consisting of those automorphisms which fix every vertex of $T \backslash T_{v}$; and for each $n$, the corresponding rigid level stabilizer $\operatorname{rist}_{G}(n)$ is the group generated by the subgroups rist ${ }_{G}(v)$ with $v$ of level $n$. In other 
words, if $\left\{v_{1}, \cdots, v_{t}\right\}$ is the $n$th layer of $T$, then

$$
\operatorname{rist}_{G}(n)=\operatorname{rist}_{G}\left(v_{1}\right) \times \cdots \times \operatorname{rist}_{G}\left(v_{t}\right) .
$$

We say that $G$ is a branch group on $T$ iff for each $n \geq 0$,

(i) $G$ acts transitively on the $n$th layer of $T$; and

(ii) $\operatorname{rist}_{G}(n)$ has finite index in $G$.

From now on, let $\left(p_{n}\right)_{n \geq 0}$ be the increasing enumeration of the primes $p \geq 5$ and let $\ell_{n}=p_{n}+1$. Let $T$ be the rooted tree of type $\left(\ell_{n}\right)_{n \geq 0}$; and for each $m \geq 0$, let $T_{m}$ be the rooted tree of type $\left(\ell_{n}\right)_{n \geq m}$. For each $n \geq 0$, let

$$
\Gamma_{n}^{0}=\operatorname{PSL}\left(2, p_{n}\right) \quad \text { and } \quad \Gamma_{n}^{1}=\operatorname{Alt}\left(\ell_{n}\right)
$$

Using the natural permutation representation of $\operatorname{PSL}\left(2, p_{n}\right)$ on the points of the projective line over $\mathbb{F}_{p_{n}}$, we can regard

$$
\operatorname{PSL}\left(2, p_{n}\right)<\operatorname{Alt}\left(\ell_{n}\right)<\operatorname{Sym}\left(\ell_{n}\right)
$$

For later use, note that the groups

$$
\left\{\operatorname{PSL}\left(2, p_{n}\right) \mid n \geq 0\right\} \cup\left\{\operatorname{Alt}\left(\ell_{n}\right) \mid n \geq 0\right\}
$$

are pairwise nonisomorphic. Let $\Delta=\operatorname{SL}(2, \mathbb{Z}[1 / 6]) * \operatorname{SL}(2, \mathbb{Z}[1 / 6])$. Then $\Delta$ is a perfect 4-generator group; and, by the proof of Segal [11, Lemma 8], for each $n \geq 0$ and $\varepsilon=0,1$, there exists a surjective homomorphism

$$
\theta_{n}^{\varepsilon}: \Delta \rightarrow \Gamma_{n}^{\varepsilon}
$$

Let $\delta_{1}, \cdots, \delta_{4}$ be a fixed set of generators of $\Delta$ and for each $n \geq 0$ and $\varepsilon=0,1$, define

$$
\alpha(i)_{n}^{\varepsilon}=\theta_{n}^{\varepsilon}\left(\delta_{i}\right) \in \Gamma_{n}^{\varepsilon}
$$

Definition 3.1. Fix some $\varphi \in 2^{\mathbb{N}}$ and for each $n \geq 0$, let

$$
S_{n}^{\varphi}=\Gamma_{n}^{\varphi(n)}=\left\langle\alpha(1)_{n}^{\varphi(n)}, \cdots, \alpha(4)_{n}^{\varphi(n)}\right\rangle
$$

Then $A_{\varphi}$ is the corresponding branch group on $T$, as in Segal [11, Lemma 1]. 
Remark 3.2. This paper has been written so as to be intelligible to readers who are unfamiliar with the details of Segal's construction. In particular, Lemma 3.4 summarizes the algebraic properties of the groups $A_{\varphi}$ that are needed in the proof of Theorem 1.3.

Definition 3.3. Let $\sigma: 2^{\mathbb{N}} \rightarrow 2^{\mathbb{N}}$ be the shift map defined by

$$
\sigma\left(\varepsilon_{0}, \varepsilon_{1}, \varepsilon_{2}, \cdots\right)=\left(\varepsilon_{1}, \varepsilon_{2}, \varepsilon_{3}, \cdots\right)
$$

If $\varphi \in 2^{\mathbb{N}}$ and $m \geq 0$, then $A_{\sigma^{m}}$ denotes the subgroup of $\operatorname{Aut}\left(T_{m}\right)$ defined in the corresponding way from the sequence $\sigma^{m} \varphi$.

In the statement of the next result, an upper composition factor of a group $G$ means a composition factor of some finite quotient $G / N$ of $G$.

Lemma 3.4. The branch group $A_{\varphi}$ satisfies the following properties.

(a) If $G \leqslant A_{\varphi}$ is a subgroup of finite index, then there exists $m \geq 0$ such that $\operatorname{rist}_{A_{\varphi}}(m) \unlhd G$.

(b) For each $m \geq 1$, the rigid level stabilizer rist $_{A_{\varphi}}(m)$ is a product of $\ell_{0} \cdots \ell_{m-1}$ copies of the subgroup $A_{\sigma^{m}} \varphi$.

(c) For each $m \geq 0$, the upper composition factors of the subgroup $A_{\sigma^{m}} \varphi$ are exactly $\left\{S_{n}^{\varphi} \mid n \geq m\right\}$.

Proof. Each of these properties is proved in Segal [11, Section 2].

At this point, it is easy to compute the Borel complexity of the isomorphism and commensurability problems for the space of groups $\left\{A_{\varphi} \mid \varphi \in 2^{\mathbb{N}}\right\}$.

Lemma 3.5. If $\varphi, \psi \in 2^{\mathbb{N}}$, then $A_{\varphi} \cong A_{\psi}$ iff $\varphi=\psi$.

Proof. This is an immediate consequence of Lemma 3.4(c).

Lemma 3.6. If $\varphi, \psi \in 2^{\mathbb{N}}$, then $A_{\varphi} \approx_{C} A_{\psi}$ iff $\varphi E_{0} \psi$.

Proof. First suppose that $\varphi E_{0} \psi$. Then there exists $m \geq 1$ such that $\sigma^{m} \varphi=\sigma^{m} \psi$. Applying Lemma 3.4(b), we see that $\operatorname{rist}_{A_{\varphi}}(m) \cong \operatorname{rist}_{A_{\psi}}(m)$ and hence $A_{\varphi} \approx_{C} A_{\psi}$.

Next suppose that $A_{\varphi} \approx_{C} A_{\psi}$; say, $G \cong H$, where $\left[A_{\varphi}: G\right],\left[A_{\psi}: H\right]<\infty$. By Lemma 3.4(a), we can suppose that $G=\operatorname{rist}_{A_{\varphi}}(m)$ for some $m \geq 0$. Hence the upper composition factors of $G$ are exactly $\left\{S_{n}^{\varphi} \mid n \geq m\right\}$. Similarly, there exists 
$t \geq 0$ such that $\operatorname{rist}_{A_{\psi}}(t) \unlhd H$ and so there exists a finite set $\mathcal{F}$ of finite simple groups such that the upper composition factors of $H$ are $\mathcal{F} \cup\left\{S_{n}^{\psi} \mid n \geq t\right\}$. Clearly if $\varphi$ is not $E_{0}$-equivalent to $\psi$, then there exists an $n$ such that $S_{n}^{\varphi} \notin \mathcal{F} \cup\left\{S_{n}^{\psi} \mid n \geq t\right\}$, which is impossible. Hence $\varphi E_{0} \psi$.

From now on, let $X \subseteq \mathcal{G}$ be the Borel subset consisting of those groups $G \in \mathcal{G}$ such that $G \approx_{C} A_{\varphi}$ for some $\varphi \in 2^{\mathbb{N}}$. Note that both $X$ and $\left\{A_{\varphi} \mid \varphi \in 2^{\mathbb{N}}\right\}$ are standard Borel spaces.

Lemma 3.7. There exists a Borel map $b: X \rightarrow\left\{A_{\varphi} \mid \varphi \in 2^{\mathbb{N}}\right\}$ such that for all $G$, $H \in X$,

(i) $G \approx_{C} b(G)$; and

(ii) if $G \cong H$, then $b(G)=b(H)$.

Proof. Let $G \in X$. Then there is a minimal $d \geq 1$ such that there exists a subgroup $H \leqslant G$ such that

(a) $[G: H]=d$; and

(b) there exists $\varphi \in 2^{\mathbb{N}}$ and $m \geq 0$ such that $H \cong \operatorname{rist}_{A_{\varphi}}(m)$.

Note that the upper composition factors of $H$ are precisely $\left\{S_{n}^{\varphi} \mid n \geq m\right\}$. Thus $H$ uniquely determines both $m$ and $\sigma^{m} \varphi$. In particular, for each such $H$, there are only finitely many possibilites for $\varphi$. Since $G$ contains only finitely many subgroups of index $d$, there are also only finitely many possibilites for $H$ and hence we can let $b(G)=A_{\varphi}$, where $\varphi$ is the lexicographically least of the finitely many elements of $2^{\mathbb{N}}$ that arise in this fashion. Clearly $b(G)$ depends only on the isomorphism class of $G$.

We are now ready to present the proof of Theorem 1.3. So suppose that $f: \mathcal{G} \rightarrow \mathcal{G}$ is a Borel reduction from $\approx_{C}$ to $\cong$ such that $f(G) \approx_{C} G$ for all $G \in \mathcal{G}$. Then $f\left(A_{\varphi}\right) \approx_{C} A_{\varphi}$ for all $\varphi \in 2^{\mathbb{N}}$. Hence, after adjusting $f$ by the map $b$ given by Lemma 3.7, we can suppose that there exists a Borel map $h: 2^{\mathbb{N}} \rightarrow 2^{\mathbb{N}}$ such that $f\left(A_{\varphi}\right)=A_{h(\varphi)}$ for all $\varphi \in 2^{\mathbb{N}}$. Now notice that if $\varphi, \psi \in 2^{\mathbb{N}}$, then

$$
\begin{array}{rlll}
\varphi E_{0} \psi & \text { iff } \quad A_{\varphi} \approx_{C} A_{\psi} & \text { (by Lemma 3.6) } \\
& \text { iff } \quad A_{h(\varphi)} \cong A_{h(\psi)} & \text { (by the assumptions on } f \text { ) } \\
& \text { iff } & h(\varphi)=h(\psi) & (\text { by Lemma } 3.5) .
\end{array}
$$


But this means that $E_{0}$ is smooth, which is a contradiction.

\section{Concluding REMARKS}

In this final section, we shall consider the analogs of Theorem 1.3 for the virtual isomorphism and quasi-isometry relations on the space $\mathcal{G}$ of finitely generated groups.

Recall that two groups $G_{1}, G_{2}$ are said to be virtually isomorphic or commensurable up to finite kernels iff there exist subgroups $N_{i} \leqslant H_{i} \leqslant G_{i}$ for $i=1,2$ satisfying the following conditions:

(a) $\left[G_{1}: H_{1}\right],\left[G_{2}: H_{2}\right]<\infty$;

(b) $N_{1}, N_{2}$ are finite normal subgroups of $H_{1}, H_{2}$ respectively; and

(c) $H_{1} / N_{1} \cong H_{2} / N_{2}$.

Let $\approx_{V I}$ be the virtual isomorphism relation on the space $\mathcal{G}$ of finitely generated groups. Then it is easily checked that $\approx_{V I}$ is a Borel equivalence relation on $\mathcal{G}$. In Thomas [12], it was shown that $(\cong)<_{B}\left(\approx_{V I}\right)$. Of course, this immediately implies that there is no Borel way of selecting an isomorphism class within each virtual isomorphism class. Alternatively, it is easy to modify the arguments of Section 3, making use of the fact that if $H$ is a subgroup of finite index in some $A_{\varphi}$, then $H$ has no nontrivial finite normal subgroups.

Next let $\approx_{Q I}$ be the quasi-isometry relation on the space $\mathcal{G}$ of finitely generated groups. (A clear account of the basic properties of the quasi-isometry relation can be found in de la Harpe [7].) It is well-known that if $G_{1}, G_{2}$ are virtually isomorphic, then $G_{1}, G_{2}$ are quasi-isometric; and it is natural to conjecture that $\left(\approx_{V I}\right)<_{B}\left(\approx_{Q I}\right)$. Of course, if true, this would immediately imply that the following two conjectures hold.

Conjecture 4.1. There does not exist a Borel way of selecting an isomorphism class within each quasi-isometry class.

Conjecture 4.2. There does not exist a Borel way of selecting a virtual isomorphism class within each quasi-isometry class. 
It seems likely that Conjecture 4.1 can be proved using a suitable modification of the arguments of Section 3. For example, for each $\tau \in 3^{\mathbb{N}}$, let $G_{\tau}$ be the corresponding Grigorchuk group, as defined in [4]. Then a routine variation of the proof of Grigorchuk [4, Theorem 7.2] yields the following analog of Lemma 3.6.

Lemma 4.3. There exists a Borel map $f: 2^{\mathbb{N}} \rightarrow 3^{\mathbb{N}}$ such that the following conditions are equivalent for all $x, y \in 2^{\mathbb{N}}$,

(a) $x E_{0} y$;

(b) $G_{f(x)}, G_{f(y)}$ are commensurable;

(c) $G_{f(x)}, G_{f(y)}$ have the same growth rate.

In particular, if $x, y \in 2^{\mathbb{N}}$, then $x E_{0} y$ iff $G_{f(x)}, G_{f(y)}$ are quasi-isometric.

Furthermore, by Nekrashevych [10, Theorem 2.10.13], the isomorphism relation on the space of groups $\left\{G_{f(x)} \mid x \in 2^{\mathbb{N}}\right\}$ is smooth and so the analog of Lemma 3.5 also holds. Unfortunately, I have been unable to prove the (presumably much more difficult) analog of Lemma 3.7 in this setting.

Finally it should be mentioned that very little is known concerning the Borel complexity of the quasi-isometry relation on the space $\mathcal{G}$ of finitely generated groups. In fact, the following result sums up the current state of knowledge regarding this problem.

Theorem 4.4 (Thomas [13]). The quasi-isometry relation on the space $\mathcal{G}$ of finitely generated groups is not smooth.

Proof. This is an immediate consequence of Lemma 4.3 .

\section{REFERENCES}

[1] C. Champetier, L'espace des groupes de type fini, Topology 39 (2000), 657-680.

[2] A. Erschler, Not residually finite groups of intermediate growth, commensurability and nongeometricity, J. Alg. 272 (2004), 154-172.

[3] J. Feldman and C. C. Moore, Ergodic equivalence relations, cohomology and von Neumann algebras, I, Trans. Amer. Math. Soc. 234 (1977), 289-324.

[4] R. I. Grigorchuk, Degrees of growth of finitely generated groups and the theory of invariant means, Math. SSSR. Izv. 25 (1985), 259-300. 
[5] R. I. Grigorchuk, Just infinite branch groups, in: M. du Sautoy, D. Segal and A. Shalev (eds), New Horizons in Pro-p Groups, Birkhäuser, Boston, 2000.

[6] R. I. Grigorchuk, Solved and Unsolved Problems Around One Group, in: L. Bartholdi, T. Ceccherini-Silberstein, T. Smirnova-Nagnibeda and A. Zuk (eds), Infinite Groups: Geometric, Combinatorial and Dynamical Aspects, Birkhäuser, Boston, 2005.

[7] P. de la Harpe, Topics in Geometric Group Theory, Chicago Lectures in Mathematics Series, The University of Chicago Press, Chicago, 2000.

[8] Harrington, L., Kechris, A. S., Louveau, A., A Glimm-Effros dichotomy for Borel equivalence relations, J. Amer. Math. Soc. 3 (1990), 903-927.

[9] S. Jackson, A.S. Kechris, and A. Louveau, Countable Borel equivalence relations, J. Math. Logic 2 (2002), 1-80.

[10] V. V. Nekrashevych, Self-similar groups, Math. Surveys and Monographs 117, Amer. Math. Soc., 2005.

[11] D. Segal, The finite images of finitely generated groups, Proc. Lond. Math. Soc. 82 (2001), 597-613.

[12] S. Thomas, The virtual isomorphism problem for finitely generated groups, Bull. Lond. Math. Soc. 35 (2003), 777-784.

[13] S. Thomas, Cayley graphs of finitely generated groups, Proc. Amer. Math. Soc. 134 (2006), 289-294.

[14] S. Thomas and B. Velickovic, On the complexity of the isomorphism relation for finitely generated groups, J. Algebra 217 (1999), 352-373.

Mathematics Department, Rutgers University, 110 Frelinghuysen Road, Piscataway, New JeRSEy 08854-8019, USA

E-mail address: sthomas@math.rutgers.edu 